A geometric examination of majorities based on difference in support

Richard Baron, Mostapha Diss, Eric Rémila, Philippe Solal 


\section{GATE Groupe d'Analyse et de Théorie Économique Lyon-St Étienne}

93, chemin des Mouilles 69130 Ecully - France

Tel. +33(0)4 72866060

Fax $+33(0) 472866090$

6, rue Basse des Rives 42023 Saint-Etienne cedex 02 - France

Tel. +33 (0)4 77421960

Fax. +33 (0)4 77421950

Messagerie électronique / Email : gate@gate.cnrs.fr

Téléchargement / Download : http://www.gate.cnrs.fr - Publications / Working Papers 


\title{
A geometric examination of majorities based on difference in support*
}

\author{
Richard Baron $^{\dagger} \quad$ Mostapha Diss $^{\dagger \ddagger} \quad$ Eric Rémila $^{\dagger} \quad$ Philippe Solal $^{\dagger}$
}

This version: May 2014

\begin{abstract}
Reciprocal preferences have been introduced in the literature of social choice theory in order to deal with preference intensities. They allow individuals to show preference intensities in the unit interval among each pair of options. In this framework, majority based on difference in support can be used as a method of aggregation of individual preferences into a collective preference: option $a$ is preferred to option $b$ if the sum of the intensities for $a$ exceeds the aggregated intensity of $b$ in a threshold given by a real number located between 0 and the total number of voters. Based on a three dimensional geometric approach, we provide a geometric analysis of the non transitivity of the collective preference relations obtained by majority rule based on difference in support. This aspect is studied by assuming that each individual reciprocal preference satisfies a $g$-stochastic transitivity property, which is stronger than the usual notion of transitivity.
\end{abstract}

KEYWORDS: Geometric voting - Reciprocal preferences - Difference in support - Stochastic transitivity.

JEL CLASSIFICATION: D72

\section{Introduction}

Given two options in a decision process, we can ask each individual if he/she prefers one option to another or if he/she is indifferent. In this case, the individual preferences are referred to as dichotomous (crisp or ordinal) preferences. In this framework, when two voters cast their votes in favor of the same option, it does not necessarily mean that the two voters share common perceptions on this option in comparison with the other option. Sen [29] is one of the first authors who points out the insufficiency of information when the individual preferences are represented by a dichotomous preference relation. The motivation for resorting to fuzzy set concepts is straightforward enough: the preference relations appear intuitively not rich enough to capture the preference intensities of decision makers. In other cases, the dichotomous preferences are too discriminating to make sense in certain decision contexts. The importance of considering intensities in the individual preferences has also been underlined by Nurmi [23, 24], Morales [22]

\footnotetext{
*For helpful comments received, the authors want to thank W.V. Gehrlein, V. Merlin, and the participants of the 13th SAET conference. Financial support by the National Agency for Research (ANR) - research program "Dynamic Matching and Interactions: Theory and Experiments" (DynaMITE) ANR. BLANC - and the "Mathématiques de la décision pour l'ingénierie physique et sociale" (MODMAD) project is gratefully acknowledged.

${ }^{\dagger}$ Université de Lyon, Lyon, F-69007, France ; CNRS, GATE Lyon Saint-Etienne, Ecully, F-69130, France ; Université Jean Monnet, Saint-Etienne, F-42000, France.

${ }^{\ddagger}$ Corresponding author: Phone: + 33 (0)4 696611 55. Email: diss@ gate.cnrs.fr.
} 
(see translation in McLean and Urken [20]), Cook and Kress [3], Meek [21] and Tanino [30], among others.

Reciprocal individual preferences have been introduced in the social choice literature for dealing with preference intensities in different fields of decision theory. Such preferences allow individuals to show preference intensities among each pair of options by a real number between 0 and 1 . Notice that, in addition to the notion of preference intensity, the representation of the individual preferences by a real number in the interval $[0,1]$ has a second different interpretation in the literature: for authors such as Orlovsky [25], Ovchinnikov [26], Basu [2], and Barrett et al. [1], among others, this number represents the degree of certainty with which the preference is held. This means that this number represents the probability that an option is judged preferable to another option for an individual. However, some authors such as Tanino [30] points out that the notions of intensity of preference and the degree of certainty are similar. In other words, a high (low) intensity is equivalent to a high (low) degree of certainty in the individual preference and vice versa.

As in the case of dichotomous preferences, transitivity is one of the most basic property required when the notion of reciprocal individual preference is introduced. This is important because we need to assume some kind of rationality condition in order to avoid the possibility of having incoherent individual preferences in election with more than two options. However, in the case of reciprocal preferences, there is no unique definition of transitivity, and many proposals have been suggested in the literature to ensure such rationality requirement (see, for instance, Dubois and Prade [7], García-Lapresta and Meneses [11], Zadeh [31], Dasgupta and Deb [4], among others). Although a variety of types of transitivity has been introduced for reciprocal relations, the notion of $g$-stochastic transitivity remains the most widely accepted and used in the literature. A general definition for $g$-stochastic transitivity is given in De Baets et al. [6] and De Baets and De Meyer [5]. Among the $g$-stochastic transitive relations, the arithmetic mean stochastic transitivity $(g=$ arithmetic mean), the weak stochastic transitivity $(g=0.5)$, the moderate stochastic transitivity $(g=\min )$, and the strong stochastic transitivity $(g=\max )$ play an important role.

Within the class of $g$-stochastic transitive reciprocal individual preferences, García-Lapresta and Llamazares [10] introduce and axiomatically characterize a new voting system as a possible method of aggregation. This new method of aggregation, called majority based on difference in support and denoted by $\widetilde{M}_{k}$, suggests that option $a$ wins against $b$ if the sum of the intensities for $a$ exceeds the sum of intensities of $b$ in a threshold $k$ given by a real number located between 0 and the total number of voters. This voting aggregation rule is based on the same idea as the one found in majority based on difference in votes $M_{k^{\prime}}$ in case preferences are dichotomous. Given two options, $a$ and $b$, a majority based on difference in votes $M_{k^{\prime}}$ indicates that $a$ is collectively preferred to $b$, when the number of individuals who prefer $a$ to $b$ exceeds the number of individuals who prefer $b$ to $a$ by at least a fixed integer $k^{\prime}$ located between 0 and the total number of voters minus 1. This rule was introduced and analyzed by García-Lapresta and Llamazares [9] and its complete axiomatic characterization can be found in Llamazares [15] and Houy [13]. Therefore, majorities based on difference in support $\widetilde{M}_{k}$ have been introduced in the social choice literature as a generalization of majorities based on difference in votes $M_{k^{\prime}}$ by considering individual intensities of preference instead of dichotomous preferences. Both rules can be interpreted as an appropriate response to the criticism concerning simple majority which requires very poor difference for declaring an option as a winner in an election. For other kinds of majorities which can be obtained through the aggregation of non dichotomous preferences and their characterization, the reader is referred to García-Lapresta [8], Llamazares and García-Lapresta [17, 18] and Llamazares [14, 16], among others.

As in other voting aggregation systems, voting paradox constitutes a key aspect of the theory. 
In this respect, Llamazares, Pérez-Asurmendi and García-Lapresta [19] provide some necessary and sufficient conditions under which $\widetilde{M}_{k}$ majorities lead to a transitive collective preference relation. These results highlight the importance of the arithmetic mean for aggregating individual intensities preferences, but also reveal that the resulting collective preferences are, in general, non transitive. The only way to obtain transitive collective preferences is to assume that $g$ is greater than or equal to the arithmetic mean and $k$ is close to the size of the population, i.e. almost unanimous support are required to avoid intransitivities.

The proofs provided by the authors are mainly algebraic. In this article, we take an alternative point of view by providing a geometric approach of the problem. The transitivity property allows us to represent the problem in the unit cube. Our study shows that the regions for which the collective preferences are non transitive are either constructed from or constitute trirectangular tetrahedrons. Therefore, we do not only get good intuitions for the impossibility results but are able to identify the shape of the regions for which the collective preference relations are non transitive. More precisely, we geometrically show that these regions evolve continuously as a function of the threshold $k$. This analysis is done for different types of $g$-stochastic transitive individual preferences.

The paper is organized as follows. In Section 2, we introduce the basic notions of reciprocal preference relations and their aggregation by mean of majorities based on difference in support. Section 3 presents the geometric examination of majorities based on difference in support, and contains the main results of the paper. As it is the rule in voting geometry, the conclusion that we draw are obtained for large electorates, i.e. when the population is assimilated to a continuum of voters. Section 4 gives a geometric insight of the problem when the population is finite as assumed in Llamazares, Pérez-Asurmendi and García-Lapresta [19].

\section{Basic framework and definitions}

In this section, the concepts of reciprocal preference relations and their aggregation in the context of majority with difference of support are introduced.

\subsection{Reciprocal preference relations}

Assume that there exist a population of $n$ voters, indexed by $i=1,2, \ldots, n$ and a finite set $X$ of $m \geq 2$ alternatives. Each voter $i$ has a reciprocal preference relation over $X$. The basic idea of a reciprocal preference relation, also called a probabilistic or an ipsodual preference relation, is that each voter provides numerical degrees of preference among the alternatives by means of real numbers $^{1}$, the intensities, within a bipolar scale in the unit interval. More specifically, for each voter $i$, a reciprocal preference over $X$ is defined as a function $Q_{i}: X \times X \rightarrow[0,1]$ such that, for each pair of alternatives $(a, b) \in X \times X, Q_{i}(a, b)+Q_{i}(b, a)=1$. The closer $Q_{i}(a, b)$ is to 1 , the more $a$ is preferred to $b$, and the closer this number is to 0 , the more $b$ is preferred to $a$. If $Q_{i}(a, b)=0.5$, then the voter $i$ is indifferent between $a$ and $b$. Notice that if, for each pair of alternatives $(a, b) \in X \times X$, we have $Q_{i}(a, b) \in\{0,1\}$, then $Q_{i}$ can be considered as an ordinal or dichotomous preference relation over $X$.

When we extend the set of alternatives from two to three alternatives, some properties of transitivity must be verified for each voter $i$ in order to guarantee individual rationality of the reciprocal preference relation. There exist various kinds of transitive properties for reciprocal preferences. Most of them are particular types of $g$-stochastic transitivity defined as follows. Let $\mathbb{G}$ be the set of continuous, commutative and non-decreasing function $g:[0.5,1] \times[0.5,1] \longrightarrow$

\footnotetext{
${ }^{1}$ Notice that in this paper we make the hypothesis that individuals vote sincerely, so that the possibility of strategic voting is not considered.
} 
$[0.5,1]$, and let $X$ be the set of alternatives. For every voter $i$, a reciprocal preference relation $Q_{i}$ on $X$ is $g$-stochastic transitive if the following condition holds for each tuple $(a, b, c) \in X \times X \times X$ :

$$
\left[Q_{i}(a, b)>0.5 \wedge Q_{i}(b, c)>0.5\right] \Rightarrow\left[Q_{i}(a, c)>0.5 \wedge Q_{i}(a, c) \geq g\left(Q_{i}(a, b), Q_{i}(b, c)\right)\right] .
$$

The most commonly $g$-stochastic transitivity relations used so far are the strong stochastic transitivity defined as $g(x, y)=\max \{x, y\}$, the moderate stochastic transitivity defined as $g(x, y)=$ $\min \{x, y\}$, the weak stochastic transitivity defined as the constant function $g(x, y)=0.5$, and the am-stochastic transitivity defined as the arithmetic mean of $x$ and $y$, i.e. $g(x, y)=(x+y) / 2$.

\subsection{Majorities based on difference in support}

Based on the reciprocal preferences expressed by the set of voters over the set of alternatives, one would like to arrive at a rule for the society that satisfies certain natural conditions. We follow in García-Lapresta and Llamazares' [10] footsteps, who introduce and axiomatically characterize majority rules based on difference in support. For this class of majority rules, denoted by $\widetilde{M}_{k}$, an alternative $a$ is collectively preferred to an alternative $b$ if the aggregated intensity of preference of $a$ over $b$ exceeds the aggregated intensity of $b$ over $a$ in a threshold $k \in[0, n[$, fixed before the election. Formally, if we denote by $P_{k}$ the asymmetric part of the ordinal collective preference over $X$ induced by $Q_{1}, \ldots, Q_{n}$ under $\widetilde{M}_{k}$, we have:

$$
\forall a, b \in X, \quad a P_{k} b: \Longleftrightarrow \sum_{i=1}^{n} Q_{i}(a, b)>\sum_{i=1}^{n} Q_{i}(b, a)+k .
$$

Using the fact that $Q_{i}(a, b)=1-Q_{i}(b, a)$ for each voter $i,(2)$ is equivalent to:

$$
\forall a, b \in X, \quad a P_{k} b: \Longleftrightarrow \sum_{i=1}^{n} Q_{i}(a, b)>\frac{n+k}{2} .
$$

The indifference part of $P_{k}$, denoted by $I_{k}$, is defined, for each pair of alternatives $(a, b) \in X \times X$, as $\neg\left(a P_{k} b\right)$ and $\neg\left(b P_{k} a\right)$. By (2), this gives:

$$
\forall a, b \in X, \quad a I_{k} b: \Longleftrightarrow\left|\sum_{i=1}^{n} Q_{i}(a, b)-\sum_{i=1}^{n} Q_{i}(b, a)\right| \leq k .
$$

Putting the relation $Q_{i}(a, b)=1-Q_{i}(b, a)$ into (4) and dividing by 2, one obtains:

$$
\forall a, b \in X, \quad a I_{k} b: \Longleftrightarrow\left|\sum_{i=1}^{n} Q_{i}(a, b)-\frac{n}{2}\right| \leq \frac{k}{2} .
$$

In order to highlight these notions, we construct an example in which three voters have to choose between three alternatives.

Example 1 Consider an election in which three voters have the following reciprocal preferences on the set of alternatives $X=\{a, b, c\}: Q_{1}(a, b)=1, Q_{1}(b, c)=1, Q_{1}(a, c)=1, Q_{2}(a, b)=0.1$, $Q_{2}(b, c)=0.7, Q_{2}(a, c)=0.2, Q_{3}(a, b)=0.8, Q_{3}(b, c)=0.1$ and $Q_{3}(a, c)=0.2$. It is easy to verify that these reciprocal preferences satisfy the strong stochastic transitivity property. For instance, voter 2 prefers $b$ to $c$ since $Q_{2}(b, c)=0.7$ and prefers $c$ to $a$ since $Q_{2}(c, a)=0.8$. The strong stochastic transitivity means that voter 2 must prefer $b$ to $a$ (i.e. $Q_{2}(b, a)>0.5$ ) and $Q_{2}(b, a)>0.8$. This is verified since $Q_{2}(b, a)=0.9$. For each pair of alternatives, the collective outcomes corresponding to these individual preferences are given in Table 1. 
Table 1: Collective outcomes in Example 1 as a function of $k$.

\begin{tabular}{rccc}
\hline & $a v s . b$ & $b v s . c$ & $a v s . c$ \\
\hline $0 \leq k<0.2$ & $a P_{k} b$ & $b P_{k} c$ & $c P_{k} a$ \\
$0.2 \leq k<0.6$ & $a P_{k} b$ & $b P_{k} c$ & $c I_{k} a$ \\
$0.6 \leq k<0.8$ & $a P_{k} b$ & $b I_{k} c$ & $c I_{k} a$ \\
$0.8 \leq k<3$ & $a I_{k} b$ & $b I_{k} c$ & $c I_{k} a$ \\
\hline
\end{tabular}

Using this framework, Llamazares et al. [19] present some necessary and sufficient conditions on the thresholds $k$ under which the majority rule $\widetilde{M}_{k}$ leads to a transitive relation $P_{k}$ defined as in (2). Their results highlight the importance of the arithmetic mean for aggregating individual reciprocal preferences. Let us summarize the three main results obtained by these authors.

Proposition 1 (Llamazares et al. [19])

(i) For each $g \in \mathbb{G}$ and each threshold $k \in[0, n-1$ [, there exist profiles of reciprocal preference relations $Q_{1}, \ldots, Q_{n}$ such that the associated collective preferences $P_{k}$ are non transitive.

(ii) For each $g \in \mathbb{G}$ greater than or equal to the arithmetic mean and each threshold $k \in$ $\left[n-1, n\left[, P_{k}\right.\right.$ is transitive for each profile of reciprocal preference relations $Q_{1}, \ldots, Q_{n}$.

(iii) For each function $g \in \mathbb{G}$ lying strictly below the arithmetic mean off the main diagonal, and each $k \in\left[n-1, n\left[\right.\right.$, there exist profiles of reciprocal preference relations $Q_{1}, \ldots, Q_{n}$ such that the associated collective preferences $P_{k}$ are non transitive.

Result (i) is an impossibility result on the set of reciprocal preference profiles. Nevertheless, the authors exhibit profiles of individual preference relations under which transitivity is satisfied. Regarding result (ii), some instances of reciprocal preference profiles are provided by the authors to show how the inconsistencies diminish when the threshold $k$ increases. Finally, result (iii) establishes that the sufficient condition given in result (ii) is also necessary. As for result (i), this result does not rule out situations in which transitivity is satisfied for some reciprocal preference profiles. Instances of such profiles are provided by the authors. These results are very instructive in so far as they reveal that inconsistencies are avoided only when the function $g$ defining the $g$-stochastic transitivity of the individual reciprocal preferences is greater than or equal to the arithmetic mean and the majority rule approaches the unanimous rule. In other words, most of the time the majority rules based on difference in support lead to impossibility results.

Llamazares et al. [19] prove their results either by constructing specific reciprocal preference relations as in results (i) and (iii) or by deriving the logical implications of the assumptions of the model as in result (ii). In this paper, we would like to take a different point of view of the problem. By using a geometric approach, we identify the shape of the regions for which $P_{k}$ is non transitive for each reciprocal preference profile and each $g$-stochastic transitivity assumption. The advantage of the geometric representation is twofold. First, we can visualize in the 3-dimensional euclidean space the regions representing the inconsistencies and its evolution as a continuous function of the parameter of the voting system when the size of the population is very large. Secondly, we can provide an alternative and simple geometric proof of the results (i), (ii) and (iii) and show how these results evolve when the population becomes large. In particular, we will be able to construct geometrically the profiles for which $P_{k}$ is non transitive for a given size of the population.

Therefore, as in Llamazares et al. [19], we study the non transitivity of the ordinal relation $P_{k}$ when the majority rule is $\widetilde{M}_{k}$ for some threshold $k$. Given three distinct alternatives $a, b$ and $c$ 
chosen in $X$, we examine the voting situation in which:

$$
a P_{k} b \wedge b P_{k} c \wedge \neg\left(a P_{k} c\right) .^{2}
$$

This means that, in addition of the well-known cyclic preferences defined as

$$
a P_{k} b \wedge b P_{k} c \wedge c P_{k} a,
$$

our examination includes the undesirable preferences defined as:

$$
a P_{k} b \wedge b P_{k} c \wedge a I_{k} c .
$$

The remaining situations include the undesirable collective preferences of the type:

$$
a P_{k} b \wedge b I_{k} c \wedge a I_{k} c .
$$

These situations are not considered in this paper. However, the examination of such undesirable cases can be done quite naturally using the same geometric approach.

In the sequel, and without loss of generality, we focus the analysis to the case of three alternatives $a, b$ and $c$. Each reciprocal preference relation $Q_{i}$ is represented by a three-dimensional vector $Q_{i}=\left(Q_{i}(a, b), Q_{i}(b, c), Q_{i}(a, c)\right)$. In order to compare easily the set of admissible individual reciprocal preference relations with the set of collective preference relations, we normalize $Q$ by computing the mean of the $Q_{i}$ and not the sum of the $Q_{i}$ as in (2)-(3). The reader can check that the two representations are equivalent (see also Llamazares et al., [19], page 8). We have:

$$
Q(a, b)=\sum_{i=1}^{n} \frac{1}{n} Q_{i}(a, b) \in[0,1], \text { and } Q(b, a)=1-Q(a, b) .
$$

Proceeding in the same manner for $(b, c)$ and $(a, c)$, we see that $Q$ is a reciprocal preference relation. Consider any pair of alternatives, say $(a, b)$, and take into account (6), we see that inequalities defining the relation $P_{k}$ in (2) and (3) and the relation $I_{k}$ in (4) and (5) can be rewritten as follows:

$$
\begin{aligned}
a P_{k} b & \Longleftrightarrow Q(a, b)>0.5+\frac{k}{2 n} . \\
a I_{k} b: \Longleftrightarrow 0.5+\frac{k}{2 n} & \geq Q(a, b) \geq 0.5-\frac{k}{2 n} .
\end{aligned}
$$

Finally, by setting $\varepsilon=k / 2 n$, the aim of this paper is to characterize the regions for which $Q$ is such that:

$$
Q(a, b)>0.5+\varepsilon \wedge Q(b, c)>0.5+\varepsilon \wedge \neg(Q(a, c)>0.5+\varepsilon) .
$$

\section{Geometrization and main results}

To our knowledge, Donald G. Saari is the first author who has shown how simple geometry tools can be used to analyze and discover new properties in voting systems where voters have ordinal preferences over a set of alternatives. Saari's geometric tools have given rise to a large number of papers. We refer the interested reader to $[27,28]$ for further information. All our results must be viewed as a characterization of the regions of inconsistencies when the population becomes

\footnotetext{
${ }^{2}$ By symmetry, we also consider the case $b P_{k} a, c P_{k} b$ and $\neg\left(c P_{k} a\right)$.
} 
arbitrarily large. This implies that the threshold $\varepsilon$ in (8) must be viewed as a share of the size $n$ of the population so that $\varepsilon$ takes its values in the interval $[0,0.5[$.

The principal advantage of the restriction to three alternatives and the representation (7) is that we can locate geometrically all possible individual and collective reciprocal preferences in a specific subset of the 3-dimensional euclidean space. Indeed, any (restricted) reciprocal preference $Q_{i}=\left(Q_{i}(a, b), Q_{i}(b, c), Q_{i}(a, c)\right)$ is located into the unit cube whose vertices are $(1,1,1),(1,0,1)$, $(0,1,1),(0,1,0),(1,0,0),(0,0,0),(1,1,0)$, and $(0,0,1)$. The six first vertices define the situations where the reciprocal preferences can be assimilated to the six linear orders and the two last vertices represent the remaining two cyclic ordinal preferences. For instance, the point $(1,0,1)$ represents the linear order given by $a \succ c \succ b$. The point $(1,1,0)$ represents the cyclic preference relation $a \succ b, b \succ c$ but $c \succ a$. Because each collective preference $Q=(Q(a, b), Q(b, c), Q(a, c))$ is defined as a convex combination of individual reciprocal preferences, it also lies in the unit cube.

Given that the function $g$ restricts the feasible individual reciprocal preferences $Q_{i}$, it also restricts its convex hull defining the feasible region of the collective preferences $Q$. We shall call this convex hull the region $R_{0}$ which is the first principal region of interest for each case of $g$-stochastic transitivity. So, keep in mind that the principal region $R_{0}$ depends on $g$. When we consider a collective preference, two exclusive cases arise: either it belongs to the region corresponding to a non transitive preference $P_{\varepsilon}$ as defined in (8), or it does not contradict the transitivity of $P_{\varepsilon}$ for the triplet under consideration. We shall call $R_{1}$ the union of the regions which guarantee the non transitivity of $P_{\varepsilon}$. Note that $R_{1}$ also depends on the type of $g$-stochastic transitivity under consideration and on the value of $\varepsilon$.

Knowing the regions $R_{0}$ and $R_{1}$, it is tempting to estimate how likely it is to observe any non transitive relation $P_{\varepsilon}$. Assuming that each collective preference $Q$ is equally probable in the feasible set of collective preferences, one could compute the ratio between the volume of $R_{1}$ and the volume of $R_{0}$ in order to estimate the probability of the non transitivity of $P_{\varepsilon}$ :

$$
\frac{\text { volume of } R_{1}}{\text { volume of } R_{0}} \text {. }
$$

Choosing the uniform distribution over all collective preferences $Q$ can be viewed as a first approximation when no information is available on the distribution of the individual reciprocal preferences. Nevertheless, this choice is disputable as soon as the underlying individual reciprocal preferences are assumed to be chosen independently according to the same distribution. Indeed, under some regular assumptions, the multivariate central limit theorem indicates that $Q$ will be distributed according to the normal distribution $\mathcal{N}\left(\mu,(\sqrt{n})^{-1} K\right)$ for $n$ sufficiently large, where $\mu$ and $K$ denote the mean and the covariance matrix of the individual distribution, respectively. Furthermore, according to the law of large numbers, $Q$ will be close to the mean of the individual distribution. Therefore, although (9) is attractive from a computational point of view, it does not fit with a probabilistic model where independence is assumed. This condition of independence is a key assumption in the two elementary models used in the framework of dichotomous preferences.

In these models, the process of determining how likely it is to observe any voting event outcome essentially involves obtaining the set of voting situations that exhibit the outcome in question, and then accumulating the likelihoods of observing the voting situations in this particular set. A voting situation indicates, for each dichotomous preference relation, the number (or the fraction) of voters associated with this preference relation. The first model, known as the Impartial Anonymous Culture condition (IAC), assumes that the voting situations are drawn independently and uniformly. The second model, known as the Impartial Culture condition (IC), is defined in terms of voter preference profiles, so that individual voter's preferences are not anonymous in voter profiles while they are anonymous in voting situations. A profile is then obtained by making independent random assignments of the possible dichotomous preference relations to 
voters, according to some probability distribution. In the IC model, this probability distribution is the uniform distribution. The voting situation that follows from this profile is then obtained by determining the number of voters that have each of the different dichotomous preference relations. It must be emphasized that no claim whatsoever is being made that either IC or IAC represents any realistic voting scenario. They are considered for two important reasons that are developed in detail in Gehrlein and Lepelley [12]. To our knowledge, there is no equivalent models in the framework of reciprocal preferences. One difficulty is that each voter may provide numerical degrees of preference among the alternatives by means of real numbers between 0 and 1 , so that the set of voting situations in not denumerable. Picking individual reciprocal preferences independently according to the same distribution as suggested above has the same spirit as IC, and so can be used to estimate how likely it is to observe any non transitive relation $P_{\varepsilon}$. In the following, we focus our analysis on the geometric properties of the region $R_{1}$.

\subsection{The case of weak stochastic transitivity}

In this section, we focus the attention on the simple case where the individual preference relations are reciprocal and $g=0.5$, i.e. the individual preference relations are weak stochastic transitive. In a first step, we delineate the feasible region $R$ of all individual reciprocal preferences inside the unit cube and the induced region $R_{0}$ of all feasible collective reciprocal preferences $Q$. We will see that $R_{0}$ is the union of $R$ and some regions in the two excluded sub-cubes for which the collective preference $Q$ is non transitive. For the sake of exposition, we cut the unit cube by the three planes given by $Q_{i}(a, b)=0.5, Q_{i}(b, c)=0.5$ and $Q_{i}(a, c)=0.5$, respectively. We get a subdivision of the unit cube into eight sub-cubes. Note that the two sub-cubes which have the vertices $(1,1,0)$ and $(0,0,1)$ respectively, must be excluded of the feasible region of all individual reciprocal preference relations whatever the $g$-stochastic transitivity function. Indeed, each point inside these two sub-cubes violates condition (1) of the $g$-stochastic transitivity. For instance, the point $Q_{i}=(0.8,0.7,0.3)$ corresponds to the situation

$$
Q_{i}(a, b)=0.8 \wedge Q_{i}(b, c)=0.7 \wedge \neg\left(Q_{i}(a, c)>0.5\right) .
$$

It follows that the region of all feasible individual reciprocal preference relations is represented by the six sub-cubes in the left-hand side of Figure 1.
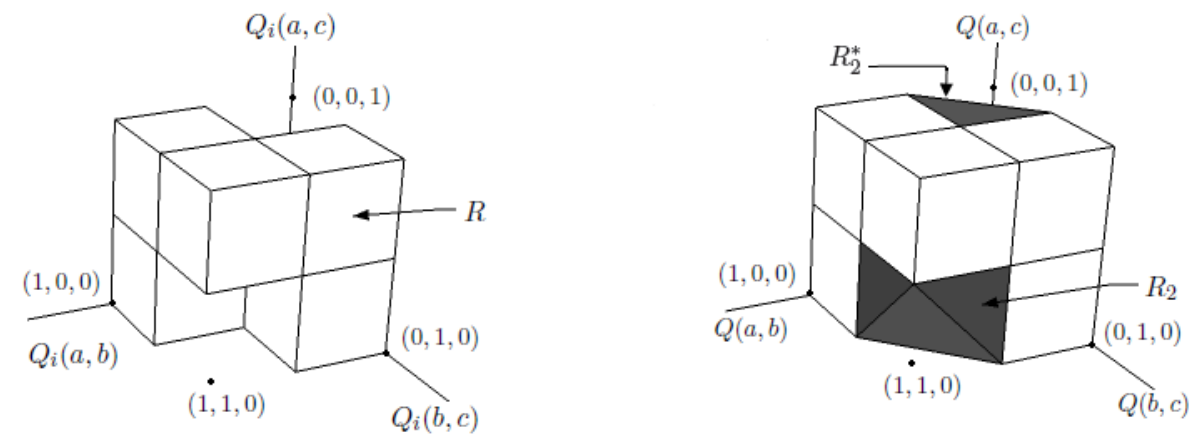

Figure 1: The entire region of all possible individual (left-hand side) and collective (right-hand side) reciprocal preferences for $g=0.5$.

We begin with the simplest case where $\varepsilon=0$. Our first result characterizes the regions of $R_{1}$ where $P_{\varepsilon}$ is non transitive for $\varepsilon=0$. Note that due to the symmetry of the problem, we only have to consider the case

$$
a P_{0} b \wedge b P_{0} c \wedge \neg\left(a P_{0} c\right) .
$$


The extension to the symmetric case

$$
b P_{0} a \wedge c P_{0} b \wedge \neg\left(c P_{0} a\right)
$$

is immediate. From a geometric point of view, if $R, H, D, G \ldots$ denote some regions on one side of the cube, the regions $R^{*}, H^{*}, D^{*}, G^{*} \ldots$ denote the symmetric regions with respect to the center of the unit cube $(0.5,0.5,0.5)$. This amounts to saying that if one proves that the collective preference $Q=(Q(a, b), Q(b, c), Q(a, c))$ is non transitive, then its image $Q^{*}=(1-Q(a, b), 1-$ $Q(b, c), 1-Q(a, c))$ obtained under this point symmetry operation, is also non transitive.

Proposition 2 For $\varepsilon=0$ and $g=0.5$, the region $R_{1}$ for which $P_{0}$ is non transitive is the union of the regions $R_{2}$ and $R_{2}^{*}$, where

$$
R_{2}=\left\{Q \in[0.5,1]^{2} \times[0,0.5]: Q(a, b)+Q(b, c)-Q(a, c) \leq 1.5\right\} .
$$

From a geometric point of view, $R_{2}$ is obtained by subtracting from the sub-cube $[0.5,1]^{2} \times[0,0.5]$ a trirectangular tetrahedron. ${ }^{3}$ The region $R_{0}$ is given by the union of $R$ and $R_{2} \cup R_{2}^{*}$.

Proof. All possible collective reciprocal preference relations $Q$ in this election lies in the region $R_{0}$ given by the convex hull of the six possible sub-cubes denoted by $R$. This convex hull is thus composed of $R$ plus some regions belonging to the two excluded sub-cubes. The entire region $R_{0}$ of all possible collective reciprocal preference relations is represented in the right-hand side of Figure 1, which consists of the six sub-cubes plus to symmetric shaded regions. We will focus the analysis on one of these two shaded regions, denoted by $R_{2}$ and $R_{2}^{*}$. Regarding $R_{2}$, its vertices $(1,1,0.5),(1,0.5,0)$ and $(0.5,1,0)$ are contained in the plane of equation given by:

$$
Q(a, b)+Q(b, c)-Q(a, c)=1.5 .
$$

So, $R_{2}$ is defined as:

$$
R_{2}=\left\{Q \in[0.5,1]^{2} \times[0,0.5]: Q(a, b)+Q(b, c)-Q(a, c) \leq 1.5\right\} .
$$

From Figure 1, one sees that $R_{2}$ can be equivalently obtained from the sub-cube $[0.5,1]^{2} \times[0,0.5]$ by subtracting a trirectangular tetrahedron where the area of its base is one-half of the area of the face of the sub-cube and its height coincides with the length of the edge of the sub-cube. Therefore, $R_{0}=R \cup R_{2} \cup R_{2}^{*}$.

When $\varepsilon>0$, several regions of interest emerge for a preference relation $P_{\varepsilon}$. These regions are added in Figure 2 (the right-hand side is an inside view). To describe each of these regions, it is useful to decompose the unit interval as follows:

$$
[0,1]=[0,0.5-\varepsilon] \cup[0.5-\varepsilon, 0.5] \cup[0.5,0.5+\varepsilon] \cup[0.5+\varepsilon, 1] .
$$

This decomposition induces a decomposition of the unit cube $[0,1]^{3}$ into 64 parallelepipeds. For instance, consider the parallelepiped $D_{3}$, in dark on Figure 2, and contained in the sub-cube $[0.5,1] \times[0,0.5] \times[0,0.5]$. It is given by:

$$
D_{3}=[0.5+\varepsilon, 1] \times[0.5-\varepsilon, 0.5] \times[0,0.5-\varepsilon] .
$$

\footnotetext{
${ }^{3}$ Recall that a trirectangular tetrahedron is a tetrahedron where all three face angles at one vertex are right angles.
} 
If we pick at random an element of $D_{3}$, then we select a collective outcome $Q$ which corresponds almost surely to a non transitive preference relation $P_{\varepsilon}$ of the following type:

$$
c P_{\varepsilon} a \wedge a P_{\varepsilon} b \wedge c I_{\varepsilon} b .
$$

The other dark parallelepipeds $D_{1}$ and $D_{2}$ lead to similar non transitive preference relations $P_{\varepsilon}$. Consider the parallelepiped $G_{2}$, in grey on Figure 2, and contained in the same sub-cube $[0.5,1]^{2} \times$ $[0,0.5]$. It is given by:

$$
G_{2}=[0.5+\varepsilon, 1] \times[0,5+\varepsilon, 1] \times[0.5-\varepsilon, 0.5] .
$$

If we pick at random an element of $G_{2}$, we select a collective outcome $Q$ which corresponds almost surely to a non transitive preference relation $P_{\varepsilon}$ of the following type:

$$
a P_{\varepsilon} b \wedge b P_{\varepsilon} c \wedge a I_{\varepsilon} c .
$$

The other grey parallelepipeds $G_{1}$ and $G_{3}$ lead to similar non transitive preference relations $P_{\varepsilon}$. Note that these preference relations $P_{\varepsilon}$ are of the same type of those associated with the $D_{1}, D_{2}$, and $D_{3}$. Consider the sub-cube $G_{0}$, in grey on Figure 2, and given by:

$$
G_{0}=[0.5+\varepsilon, 1]^{2} \times[0,0.5-\varepsilon] .
$$

It contains only elements corresponding to cyclic preferences $P_{\varepsilon}$. Consider now the parallelepiped $\mathrm{H}_{2}$, hatched on Figure 2, and contained in the sub-cube $[0.5,1]^{2} \times[0,0.5]$. It is given by:

$$
H_{2}=[0.5,0.5+\varepsilon] \times[0.5+\varepsilon, 1] \times[0.5-\varepsilon, 0.5] .
$$

If we pick at random an element of $H_{2}$, we select a collective outcome $Q$ which corresponds almost surely to a preference relation $P_{\varepsilon}$ of the following type:

$$
a I_{\varepsilon} b \wedge b P_{\varepsilon} c \wedge c I_{\varepsilon} a .
$$

These preference relations $P_{\varepsilon}$ do not satisfy (8), and so are not considered as non transitive. The other hatched parallelepipeds $H_{1}$ and $H_{3}$ lead to similar preference relations $P_{\varepsilon}$. Finally, the subcube $W$, in white in the right-hand side of Figure 2, is given by:

$$
W=[0.5,0.5+\varepsilon]^{2} \times[0.5-\varepsilon, 0.5] .
$$

$W$ contains collective outcomes which correspond almost surely to preference relations $P_{\varepsilon}$ of the following type:

$$
a I_{\varepsilon} b \wedge b I_{\varepsilon} c \wedge a I_{\varepsilon} c .
$$

Once again, these preference relations do not satisfy (8), and as such are not considered as non transitive. All these regions must be taken into consideration when we characterize the region $R_{1}$ for which $P_{\varepsilon}$ is non transitive. The following proposition exploits these facts.
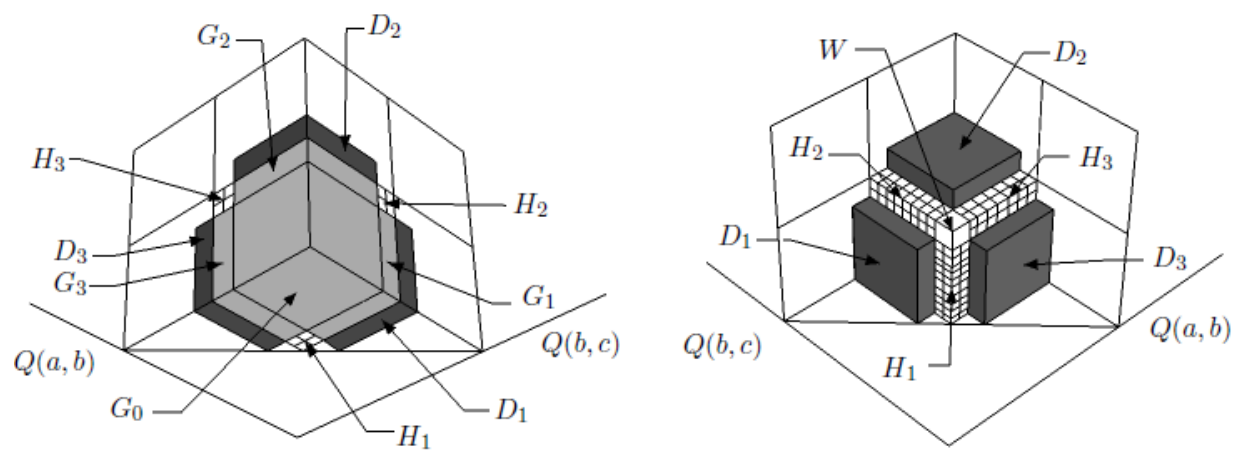

Figure 2: Some regions of interest for $P_{\varepsilon}$. The right-hand side is an inside view of the situation. 


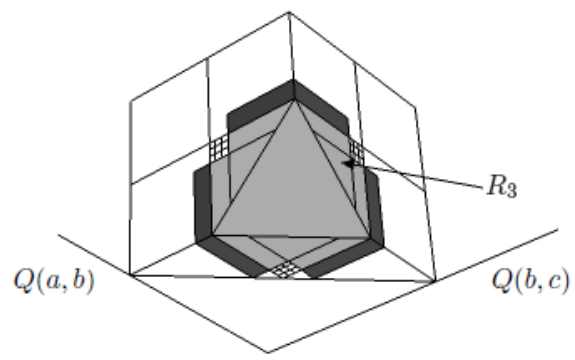

Figure 3: The region for which $P_{\varepsilon}$ is non transitive for $g=0.5$ and $\left.\left.\varepsilon \in\right] 0,0.25\right]$.

In the following proposition and for the rest of the article, $D$ and $H$ are defined as:

$$
D=\bigcup_{l=1}^{3} D_{l}, \quad H=\bigcup_{l=1}^{3} H_{l} .
$$

Proposition 3 For $g=0.5$, the region $R_{1}$ for which $P_{\varepsilon}$ is non transitive is the union of the regions $R_{3}$ and $R_{3}^{*}$ where $R_{3}=D \cup\left[R_{2} \backslash(H \cup W)\right]$. The shape of the region $R_{2} \backslash(H \cup W)$ is a function of $\varepsilon$ :

1. For $\varepsilon \in] 0,0.25],(H \cup W) \subseteq R_{2}$;

2. For $\varepsilon \in] 0.25,1 / 3], W \subseteq R_{2}$ and, for each $l \in\{1,2,3\}, H_{l} \backslash R_{2}$ is a trirectangular tetrahedron.

3. For $\varepsilon \in] 1 / 3,0.5\left[\right.$, for each $l \in\{1,2,3\},\left(H_{l} \cup W\right) \backslash R_{2}$ and $W \backslash R_{2}$ are trirectangular tetrahedrons and

$$
\bigcap_{l=1}^{3}\left(H_{l} \cup W\right) \backslash R_{2}=W \backslash R_{2} .
$$

Proof. Because $g=0.5$, the region $R_{0}$ is still the region represented in the right-hand side of Figure 1. The region $R_{1} \subseteq R_{0}$ is formed by two symmetric connected regions. One of these two connected regions, denoted by $R_{3}$, is represented by the dark and grey regions on Figure 3. By symmetry of the problem, the second region $R_{3}^{*}$ is similar to $R_{3}$, but located on the other side of the cube. The union of these regions defines the region $R_{1}$. We decompose $R_{3}$ as follows: the three dark regions $D_{1}, D_{2}$ and $D_{3}$ plus $R_{2} \cap R_{1}$, where $R_{2}$ is given by Proposition 2. So, it remains to characterize $R_{2} \cap R_{1}$. Note that $R_{2}=\left(R_{2} \cap R_{1}\right) \cup\left(R_{2} \backslash R_{1}\right)$. As we already know $R_{2}$, let us compute $R_{2} \backslash R_{1}$. From the discussion preceding Proposition 3, we deduce that:

$$
R_{2} \backslash R_{1}=\left(H_{1} \cap R_{2}\right) \cup\left(H_{2} \cap R_{2}\right) \cup\left(H_{3} \cap R_{2}\right) \cup\left(W \cap R_{2}\right) .
$$

It follows that:

$$
\begin{aligned}
R_{3} & =D \cup\left(R_{2} \cap R_{1}\right) \\
& =D \cup\left(R_{2} \backslash\left(R_{2} \backslash R_{1}\right)\right) \\
& =D \cup\left[R_{2} \backslash\left(\left(H \cap R_{2}\right) \cup\left(W \cap R_{2}\right)\right)\right] . \\
& =D \cup\left[R_{2} \backslash(H \cup W)\right] .
\end{aligned}
$$

In order to delineate the region $R_{3}$, we distinguish three cases according to the value taken by $\varepsilon$. Our cases are issued from the structure of the intersections appearing above, i.e. the relative 
position of the plane of equation (10) delimiting the region $R_{2}$ and the regions $W, H_{1}, H_{2}, H_{3}$. The region $R_{3}$ evolves continuously as a function of $\varepsilon$ and its geometric representation changes from case to case.

Case 1: $\varepsilon \in] 0,0.25]$. Figure 3 represents an instance of the problem for $\varepsilon \in] 0,0.25]$. Indeed, when $\varepsilon \in] 0,0.25], W$, and $H_{1}, H_{2}, H_{3}$ are contained in $R_{2}$. To see this, it suffices to note that each point of these regions lies above the plane of equation (10). To compute the critical value 0.25 , consider, without loss of generality, the hatched parallelepiped $\mathrm{H}_{2}$. Pick the vertex of coordinates $(0.5+\varepsilon, 1,0.5-\varepsilon)$. By (10):

$$
0.5+\varepsilon+1-0.5+\varepsilon \leq 1.5 \text { if and only if } \varepsilon \in] 0,0.25] .
$$

In a similar way, the vertex $(0.5+\varepsilon, 0.5+\varepsilon, 0.5-\varepsilon)$ of the white cube $W$ lies above the plane of equation (10) if and only if $\varepsilon \in[0,1 / 3]$. Therefore, $(H \cup W) \subseteq R_{2}$.

Case 2: $\varepsilon \in] 0.25,1 / 3]$. In this case, the white cube $W$ is still contained in $R_{2}$ whereas the hatched parallelepipeds $H_{1}, H_{2}, H_{3}$ are not longer contained in $R_{2}$. The critical value $\varepsilon=1 / 3$ is obtained when $W$ intersects the plane of equation (10). This situation is illustrated in the left-hand side of Figure 4. Therefore, $W \subseteq R_{2}$ but $H_{l} \backslash R_{2}$ is non empty for each $l \in\{1,2,3\}$. To determine the shape of each polyhedron $H_{l} \backslash R_{2}, l \in\{1,2,3\}$, let us focus, without loss of generality, on the hatched parallelepiped $H_{2}$. The plane of equation (10) cuts $H_{2}$ into two parts such that each vertex of $H_{2}$ is an element of $R_{2}$ except the vertex of coordinates $(0.5+\varepsilon, 1,0.5-\varepsilon)$. It follows that $H_{2} \backslash R_{2}$ is a trirectangular tetrahedron. For the sake of completeness, let us locate the vertices of the trirectangular tetrahedron $H_{2} \backslash R_{2}$. All three face angles at $Q^{0}=(0.5+\varepsilon, 1,0.5-\varepsilon)$ are right angles, and the three edges $\left[Q^{0} Q^{1}\right],\left[Q^{0} Q^{2}\right]$ and $\left[Q^{0} Q^{3}\right]$ that meet at the right angle are the legs of the tetrahedron. The coordinates of the points $Q^{1}, Q^{2}$ and $Q^{3}$ are contained in the plane of equation (10) (see Figure 4). The coordinates of $Q^{3}$ are $Q^{3}(a, b)=0.5+\varepsilon, Q^{3}(a, c)=0.5-\varepsilon$ and by (10) we find $Q^{3}(b, c)=1.5-2 \varepsilon$. Proceeding in a similar way for the points $Q^{1}$ and $Q^{2}$, we find that $Q^{1}=(1-\varepsilon, 1,0.5-\varepsilon)$ and $Q^{2}=(0.5+\varepsilon, 1, \varepsilon)$. From these coordinates, we find that the length of each leg is equal to $(2 \varepsilon-0.5)$. It follows that each of these tetrahedrons grows as a function of $\varepsilon$. Beyond the critical value $\varepsilon=1 / 3$, these trirectangular tetrahedrons intersect each other to form a new tetrahedron. This situation is illustrated in the right-hand side of Figure 4, and studied in Case 3. Note these tetrahedrons correspond to collective preferences such that: they are not considered as non transitive according to (8), and they cannot be obtained by a convex combination of individual reciprocal preferences.

Case 3: $\varepsilon \in[1 / 3,0.5[$. Beyond the critical value $\varepsilon=1 / 3$, the trirectangular tetrahedrons intersect each other as illustrated in the right-hand side of Figure 4. To better understand the geometric form taken by this intersection, it is interesting to consider again the inside view of our problem as depicted by the right-hand side of Figure 2. As $\varepsilon$ increases, the white cube $W$ grows as well. The regions $H_{1} \backslash R_{2}, H_{2} \backslash R_{2}$ and $H_{3} \backslash R_{2}$ do no longer constitute trirectangular tetrahedrons when the plane of equation (10) cuts $W$ into two parts, i.e. when $\varepsilon>1 / 3$. This is true because the plane of equation (10) cuts each $H_{l} \backslash R_{2}$ in such a way that at least two vertices remain in each side of this plane. On the contrary, by considering the parallelepipeds $\left(H_{l} \cup W\right), l \in\{1,2,3\}$, we get that the regions $\left(H_{1} \cup W\right) \backslash R_{2},\left(H_{2} \cup W\right) \backslash R_{2},\left(H_{3} \cup W\right) \backslash R_{2}$ and $W \backslash R_{2}$ are four trirectangular tetrahedrons. Furthermore, we have:

$$
\bigcap_{l=1}^{3}\left(H_{l} \cup W\right) \backslash R_{2}=W \backslash R_{2} .
$$

Proceeding in a similar fashion as in Case 2, we obtain that the length $3 \varepsilon-1$ of each leg of the trirectangular tetrahedron $W \backslash R_{2}$ is an increasing function of $\varepsilon$. Note also that the legs of the tetrahedron $\left(H_{l} \cup W\right) \backslash R_{2}, l \in\{1,2,3\}$, can be computed in a manner similar to those of $H_{l} \backslash R_{2}$ in Case 2. This completes the proof of Proposition 3. 

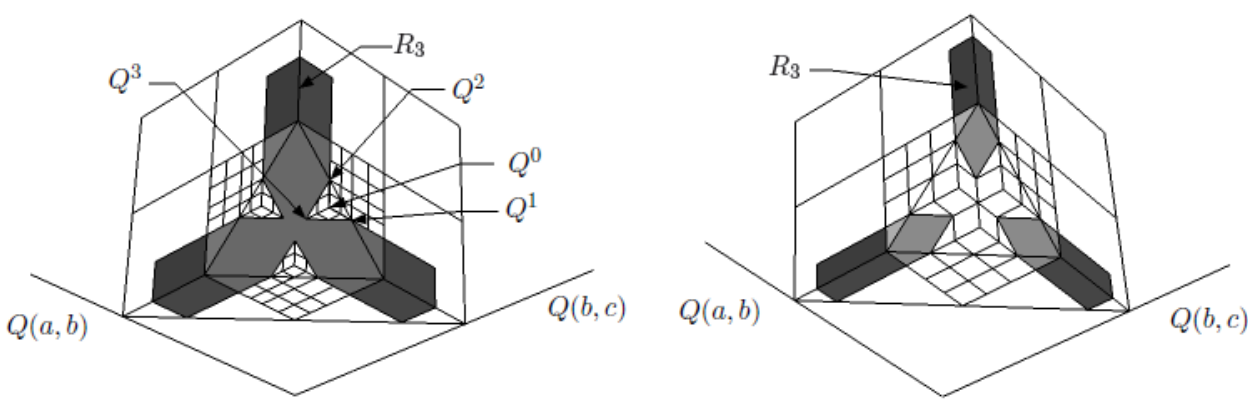

Figure 4: The region for which $P_{\varepsilon}$ is non transitive for $g=0.5$ and $\left.\left.\varepsilon \in\right] 0.25,1 / 3\right]$ (left-hand side) and for $g=0.5$ and $\varepsilon \in] 1 / 3,0.5]$ (right-hand side).

Thanks to Propositions 2 and 3, the geometric construction of the polyhedrons $R_{2}$ and $R_{3}$ can be presented in a simple way. For $\varepsilon=0, R_{2}$ is obtained from the sub-cube $[0.5,1]^{2} \times[0,0.5]$ by subtracting a tetrahedron. For small values of $\varepsilon, R_{3}$ is the union of $D$ and $R_{2} \backslash(H \cup W)$ such that $(W \cup H) \subseteq R_{2}$. For intermediate values of $\varepsilon$, the cube $W$ is still contained in $R_{2}$, but one part of the region $H$ lies outside $R_{2}$. This is consequence of the fact that the regions $H$ and $W$ grow continuously in $\varepsilon$. It appears that $H \backslash R_{2}$ is a trirectangular tetrahedron. For high values of $\varepsilon$, part of the cube $W$ lies outside $R_{2}$. The region $W \backslash R_{2}$ forms also a trirectangular tetrahedron. Therefore, the region for which $P_{\varepsilon}$ is non transitive on one side of the unit cube grows continuously in $\varepsilon$, evolving from $R_{2}$ to $D \cup\left[R_{2} \backslash(H \cup W)\right]$ when $\varepsilon$ varies from 0 to 0.5 . Similar arguments apply to the other side of the unit-cube.

\subsection{A sub-class of $g$-stochastic transitivity relations}

In the previous section, we have studied the case of weak stochastic transitivity, which amounts to choosing the smallest function $g$ in $\mathbb{G}$. Now, consider any $g$ in $\mathbb{G}$. Because $g\left([0.5,1]^{2}\right) \subseteq[0.5,1]$, one deduces that the vertex $(1,1,1)$ of the unit cube corresponding to the linear order $Q_{i}(a, b)=$ $Q_{i}(b, c)=Q_{i}(a, c)=1$ belongs to the feasible region $R$ of all individual reciprocal preferences. Proceeding in the same way for each sub-cube, one deduces that the six vertices corresponding to the six linear orders belong to the feasible region $R$ of all individual reciprocal preferences. It follows that the convex hull $C$ of these six vertices is contained in the convex hull $R_{0}$ of $R$ : $C \subseteq R_{0}$. To compute $C$, consider its vertices $(1,1,1),(1,0,0)$ and $(0,1,0)$. They are contained in the plane of equation given by:

$$
Q(a, b)+Q(b, c)-Q(a, c)=1 .
$$

By a symmetry argument, one obtains:

$$
C=\left\{Q \in[0,1]^{3}: 0 \leq Q(a, b)+Q(b, c)-Q(a, c) \leq 1\right\} .
$$

Select the sub-class $\mathbb{G}^{0}$ of functions $g:[0.5,1]^{2} \longrightarrow[0.5,1]$ in $\mathbb{G}$ such that:

$$
g(Q(a, b), Q(b, c)) \geq Q(a, b)+Q(b, c)-1,
$$

where $Q$ is a point lying in the sub-cube $[0.5,1]^{3}$. Applying this condition in each sub-cube, one obtains that $R \subseteq C$, and so $R_{0} \subseteq C$, which yields, by the above arguments, $C=R_{0}$. It follows that, for a fixed $\varepsilon$, the region $R_{1}$ of non transitivity of $P_{\varepsilon}$ is the same whatever $g \in \mathbb{G}^{0}$. Among the functions $g$ belonging to $\mathbb{G}^{0}$, we found the arithmetic mean, the maximum function and the 
minimum function. In the rest of this section, we will focus on this sub-class $\mathbb{G}^{0}$ of functions, so that the results obtained are valid in particular for the am-stochastic transitivity relation, the strong transitivity relation and the moderate transitivity relation.

As an example, consider Figure 5. The left-hand side of this figure depicts for three sub-cubes the faces (in dark) delimiting the feasible regions of the individual reciprocal preference relations satisfying the condition of moderate stochastic transitivity. The right-hand side of this figure depicts for the same three sub-cubes the faces delimiting the feasible regions of the individual reciprocal preference relations satisfying the condition of strong stochastic transitivity. These faces represent the minimum (left-hand side) and maximum (right-hand side) operators on three subcubes. For each of these three sub-cubes, the feasible reciprocal preference relations are located behind these faces. The same approach can be used for the arithmetic mean function. The left-hand side of Figure 6 depicts for three sub-cubes the faces (in dark) delimiting the feasible regions of the individual reciprocal preference relations satisfying the condition of arithmetic mean stochastic transitivity. The important point to notice is that in each case the individual reciprocal preference relations defined by the coordinates $(1,1,1),(1,0,0)$ and $(0,1,0)$ are feasible. It follows easily from this that the convex hull of each of these three sets of feasible reciprocal preference relations coincides, i.e. the region $R_{0}$ is the same when $g$ is defined as the maximum, the minimum or the arithmetic mean. The region $R_{0}$ is represented in the right-hand side of Figure 6, where the dark triangle is the face of $R_{0}$ contained in the plane of equation (16).
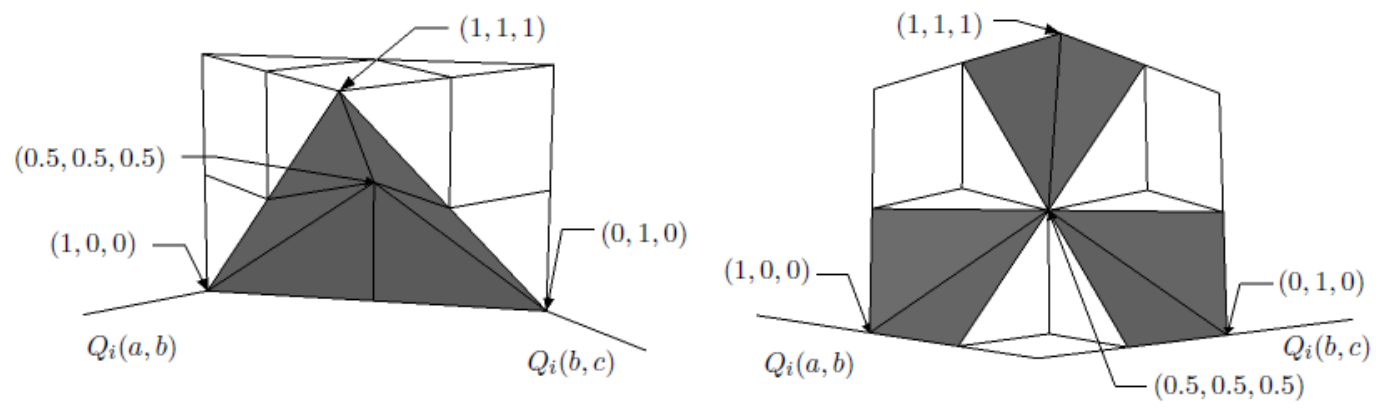

Figure 5: Regions of feasible individual reciprocal preference relations for $g=\min$ (left-hand side) and $g=\max$ (right-hand side).
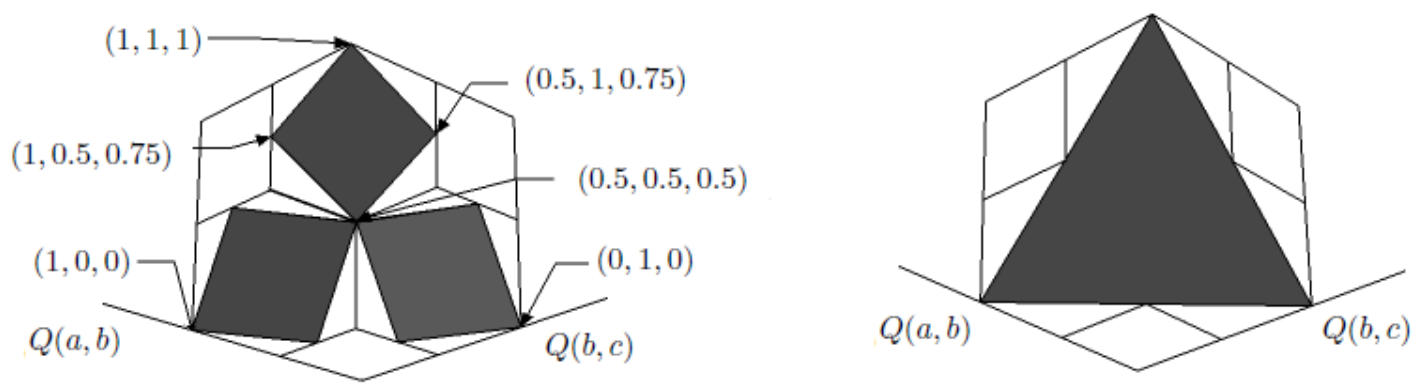

Figure 6: Regions of feasible individual reciprocal preference relations for $g=a m$ (left-hand side) and the region $R_{0}$ of all possible collective reciprocal preference relations for the cases of $g$-stochastic relations where $g \in \mathbb{G}^{0}$ (right-hand side).

From the above discussion, we obtain the following result. 
Proposition 4 For $\varepsilon=0$ and $g \in \mathbb{G}^{0}$, the region $R_{1}$ for which $P_{0}$ is non transitive is the union of the regions $R_{4}$ and $R_{4}^{*}$, where $R_{4}$ is a trirectangular tretrahedron whose vertices are given by $(0.5,1,0.5)(0.5,0.5,0),(1,0.5,0.5)$ and the center of the unit cube $(0.5,0.5,0.5)$. The region $R_{0}$ is given by $C$.

Proof. As mentioned above $C=R_{0}$ for each $g \in \mathbb{G}^{0}$. From Figure 6, remark that $R_{0}$ is obtained by subtracting from the unit cube two trirectangular tetrahedrons with unit legs. The region $R_{1}$ is the part of the region $R_{0}$ contained in the two excluded sub-cubes of the unit cube. One-half of this region $R_{1}$, denoted by $R_{4}$, is represented in the left-hand side of Figure 7 . Consider the excluded sub-cube $[0.5,1]^{2} \times[0,0.5]$. Its vertices $(0.5,1,0.5)(0.5,0.5,0),(1,0.5,0.5)$ are contained in the plane of equation given by

$$
Q(a, b)+Q(b, c)-Q(a, c)=1,
$$

and delimiting $C$. One easily verifies that its vertex $(0.5,0.5,0.5)$ belongs to $C$, whereas the four other vertices lie outside $C$. It follows that $R_{4}=C \cap\left([0.5,1]^{2} \times[0,0.5]\right)$ forms a trirectangular tetrahedron with legs of length 0.5 , and whose vertices are $(0.5,1,0.5)(0.5,0.5,0),(1,0.5,0.5)$ and the center of unit cube $(0.5,0.5,0.5)$ (see the left- hand side of Figure 7 ).
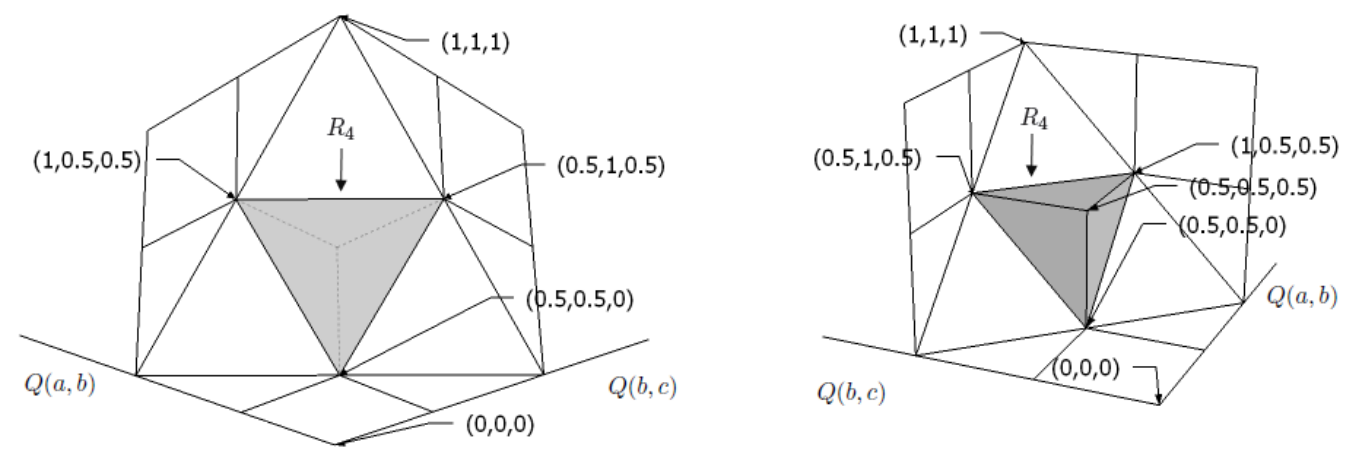

Figure 7: The region for which $P_{\varepsilon}$ is non transitive for $\varepsilon=0$ and $g \in \mathbb{G}^{0}$. The right-hand side of this figure provides an inside view of this region.

In the following, $G$ is defined as:

$$
G=\bigcup_{l=1}^{3} G_{l} .
$$

Proposition 5 For $g \in \mathbb{G}^{0}$, the region $R_{1}$ for which $P_{\varepsilon}$ is non transitive is the union of the regions $R_{5}$ and $R_{5}^{*}$ where $R_{5}=\left(G_{0} \cup G \cup D\right) \cap R_{0}$, and $R_{0}=C$. The shape of the region $R_{5}$ is a function of $\varepsilon$ :

1. For $\varepsilon \in] 0,1 / 6], G_{0} \cap R_{0} \neq \emptyset, G \cap R_{0} \neq \emptyset$, and $D \cap R_{0} \neq \emptyset$. Furthermore, for each $l \in\{1,2,3\},\left(G_{0} \cup G_{l} \cup D_{l}\right) \cap R_{0}$ is a trirectangular tetrahedron. The intersection between these trirectangular tetrahedrons constitutes also a trirectangular tetrahedron contained in $G_{0}$.

2. For $\varepsilon \in] 1 / 6,0.25\left[, G_{0} \cap R_{0}=\emptyset, G \cap R_{0} \neq \emptyset\right.$, and $D \cap R_{0} \neq \emptyset$. Furthermore, the polyhedrons $\left(G_{l} \cup D_{l}\right) \cap R_{0}, l \in\{1,2,3\}$, form three pairwise-disjoint trirectangular tetrahedrons. 
3. For $\varepsilon \in\left[0.25,0.5\left[, G_{0} \cap R_{0}=\emptyset, G \cap R_{0}=\emptyset\right.\right.$, and $D \cap R_{0} \neq \emptyset$. Furthermore, the polyhedrons $D_{l} \cap R_{0}, l \in\{1,2,3\}$, form also a collection of pairwise-disjoint trirectangular tetrahedrons.

Proof. The region $R_{0}$ still coincides to $C$. The region $R_{1}$ is constituted by the dark and grey regions, represented in Figure 2, contained in the region $R_{0}$ represented in Figure 6. To delineate the region $R_{1}$, formed by two symmetric and connected regions, denoted by $R_{5}$ and $R_{5}^{*}$ respectively, we need to consider the following regions already used in the previous results and represented in Figure 2: the sub-cube $G_{0}$ and the parallelepipeds $G_{1} \cup D_{1}, G_{2} \cup D_{2}$ and $G_{3} \cup D_{3}$. Each of these parallelepipeds share a face with $G_{0}$. The region $\left(G_{0} \cup G \cup D\right) \cap R_{0}$ constitutes one of the two symmetric and connected regions of $R_{1}$. Thus, we have:

$$
R_{5}=\left(G_{0} \cap R_{0}\right) \cup\left(G \cup D \cap R_{0}\right)=\left(G_{0} \cup G \cup D\right) \cap R_{0} .
$$

In order to characterize the shape of $R_{1}$, we distinguish several cases according to the value taken by $\varepsilon$. Our cases are issued from the structure of the intersections appearing above, i.e. the relative position of the plane of equation (16) delimiting one connected part of the region $R_{0}$ and the regions $G_{0}, G_{1} \cup D_{1}, G_{2} \cup D_{2}$ and $G_{3} \cup D_{3}$. The region $R_{1}$ evolves continuously as a function of $\varepsilon$ and its geometric representation changes from case to case.

Cases 2 and 3: $\varepsilon \in] 1 / 6,0.5]$. Consider the sub-cube $G_{0}=[0.5+\varepsilon, 1]^{2} \times[0,0.5-\varepsilon]$. Using the plane of equation (16) we see that $G_{0} \cap R_{2}=\emptyset$. Indeed, take the vertex $(0.5+\varepsilon, 0.5+\varepsilon, 0.5-\varepsilon)$ of $G_{0}$. By (16), we have:

$$
0.5+\varepsilon+0.5+\varepsilon-0.5+\varepsilon>1 \text { if and only if } \varepsilon>1 / 6 .
$$

The region $R_{5}=(G \cup D) \cap R_{0}$ is represented on Figure 8 for $\varepsilon=0.25$. More precisely, $R_{5}$ corresponds to the three dark tetrahedrons in the right-hand side of Figure 8. The right-hand side of Figure 8 gives an inside view of these tetrahedrons only. The white triangles of the left-hand side of Figure 8 are the faces of these tetrahedrons lying on the plane of equation (16). To understand this point, consider, without loss of generality, the polyhedron $\left(G_{2} \cup D_{2}\right) \cap R_{0}$. The parallelepiped $G_{2} \cup D_{2}$ is given by $[0.5+\varepsilon, 1]^{2} \times[0.5-\varepsilon, 0.5+\varepsilon]$. The vertices $(1,0.5+\varepsilon, 0.5+\varepsilon)$ and $(0.5+\varepsilon, 1,0.5+\varepsilon)$ of this parallelepiped are contained in the plane of equation (16). Furthermore, the vertex of coordinates $(0.5+\varepsilon, 0.5+\varepsilon, 0.5+\varepsilon)$ of this parallelepiped lies above the plane of equation (16). Finally, among the points of coordinates $(0.5+\varepsilon, 0.5+\varepsilon, Q(a, c))$ belonging to $R_{0}$, the only one contained in the plane of equation (16) is the point of coordinates $(0.5+\varepsilon, 0.5+\varepsilon, 2 \varepsilon)$. The convex hull of these four points contained in $R_{0}$ forms a trirectangular tetrahedrons with legs of length $0.5-\varepsilon$. Proceeding in the same way for $\left(G_{1} \cup D_{1}\right) \cap R_{0}$ and $\left(G_{3} \cup D_{3}\right) \cap R_{0}$, conclude that $(G \cup D) \cap R_{0}$ is the union of three pairwise-disjoint trirectangular tetrahedrons. Note that when $\varepsilon \in] 0.25,0.5]$, the regions $G_{1} \cap R_{0}, G_{2} \cap R_{0}$ and $G_{3} \cap R_{0}$ are empty, as claimed in Case 3. This is also the reason why the tetrahedrons are colored in dark in the right hand side of Figure 8. In Figure 9, $\varepsilon=1 / 6$ so that $G_{1} \cap R_{0}, G_{2} \cap R_{0}$ and $G_{3} \cap R_{0}$ are non empty.

Case 1: $\varepsilon \in] 0,1 / 6]$. The left-hand side of Figure 10 provides an outside view of the situation where $\varepsilon \in] 0,1 / 6]$. As in Figure 8, the faces of the parallelepipeds are supposed to be transparent in order to make visible the intersections between the plane of equation (16) and these parallelepipeds. The right-hand side of Figure 10 provides an inside view of such a situation. We see that the intersection between the three trirectangular tetrahedrons forms a new trirectangular tetrahedron contained in $G_{0}$. When $\varepsilon$ decreases, the cube in grey $G_{0}$ grows. The regions $\left(G_{1} \cup D_{1}\right) \cap R_{0}$, $\left(G_{2} \cup D_{2}\right) \cap R_{0}$ and $\left(G_{3} \cup D_{3}\right) \cap R_{2}$ do no longer constitute trirectangular tetrahedrons when the plane of equation (16) cuts $G_{0}$ into two parts, i.e. when $\varepsilon<1 / 6$. By contrast, the regions $\left(G_{1} \cup D_{1} \cup G_{0}\right) \cap R_{0},\left(G_{2} \cup D_{2} \cup G_{0}\right) \cap R_{0},\left(G_{3} \cup D_{3} \cup G_{3}\right) \cap R_{0}$ and $G_{0} \cap R_{0}$ constitute four 
trirectangular tetrahedrons. The arguments are similar to those of Case 3 contained in the proof of Proposition 3.

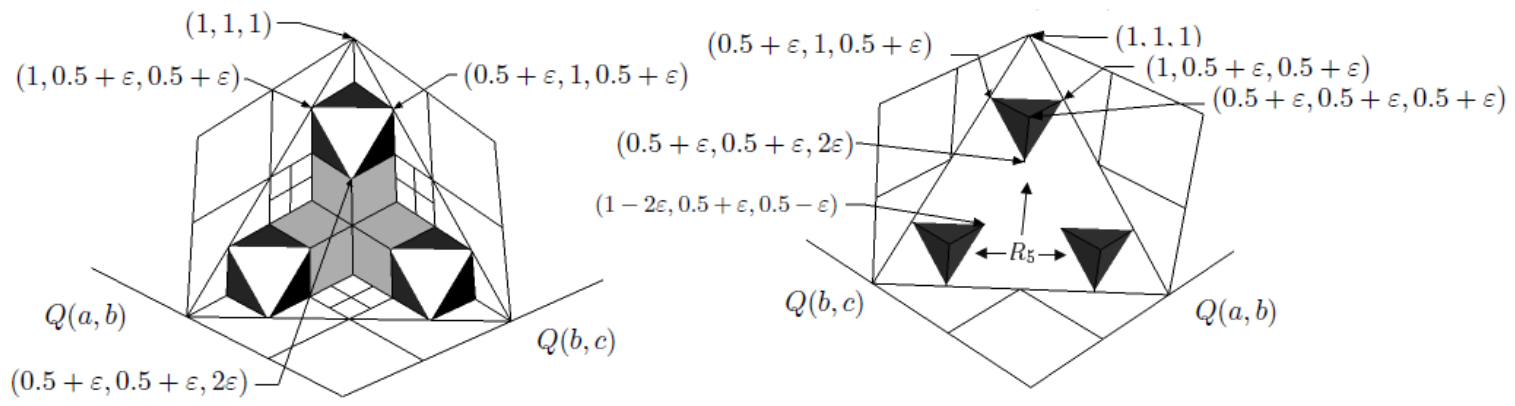

Figure 8: The region for which $P_{\varepsilon}$ is non transitive for $g \in \mathbb{G}^{0}$ and $\varepsilon \in[1 / 6,0.5[$. This figure is drawn for $\varepsilon=1 / 4$. The figure in the right-hand side is an inside view of this region.
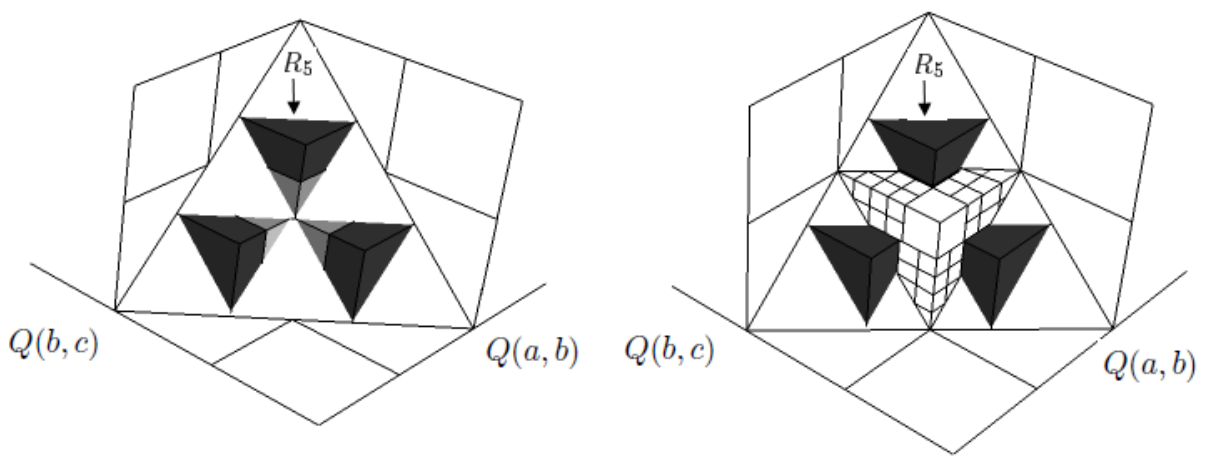

Figure 9: The region for which $P_{\varepsilon}$ is non transitive for $g \in \mathbb{G}^{0}$ and $\varepsilon=1 / 6$. The figure in the right-hand side is a full inside view of the unit cube.
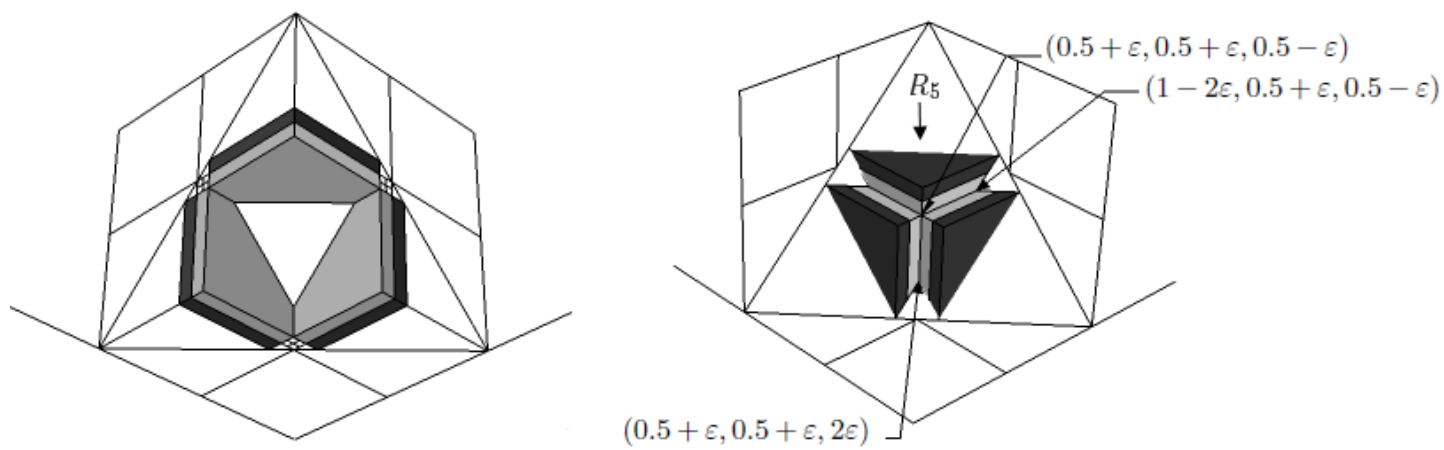

Figure 10: The region for which $P_{\varepsilon}$ is non transitive for $g \in \mathbb{G}^{0}$ and $\left.\left.\varepsilon \in\right] 0,1 / 6\right]$. The figure in the right-hand side is an inside view of this region.

Propositions 4 and 5 allow to understand the geometric construction of the polyhedrons $R_{4}$ and $R_{5}$. For high values of $\varepsilon, R_{5}$ is defined as the intersection between the polyhedron $D$ and the 
region $R_{0}$. The parallelepipeds $D_{l}, l \in\{1,2,3\}$, have a non empty intersection with $R_{0}$ whereas the parallelepipeds $G_{l}, l \in\{1,2,3\}$, lie outside $R_{0}$. The latter is obtained from the unit cube by subtracting a trirectangular tetrahedron with unit length legs. The intersection between each parallelepiped $D_{l}, l \in\{1,2,3\}$, and $R_{0}$ is itself a trirectangular tetrahedron. For intermediate values of $\varepsilon$, one region of each parallelepiped $G_{l}, l \in\{1,2,3\}$, lies inside $R_{0}$, which explains the transition from Case 2 to Case 3, even if the shape of the regions of interest does not change: because $G_{l}$ and $D_{l}$ have a common face, we still obtain a trirectangular tetrahedron when we consider the intersection $R_{0} \cap\left(G_{l} \cap D_{l}\right)$. The regions $R_{0} \cap\left(G_{l} \cap D_{l}\right), l \in\{1,2,3\}$, are pairwise disjoint up to $\varepsilon=1 / 6$. At this value, they meet at the point $(2 / 3,2 / 3,1 / 3)$, which is contained in the plane of equation (16) defining the region $R_{0}$. Below this value, the intersection between these tetrahedrons forms a new tetrahedron contained in $G_{0}$. When $\varepsilon$ vanishes, this new tetrahedron converges to $R_{4}$. Therefore, the region for which $P_{\varepsilon}$ is non transitive on one side of the unit cube grows continuously as a function of $\varepsilon$, evolving from $D \cap R_{0}$ to $R_{4}$ when $\varepsilon$ varies from 0.5 to 0 . Similar arguments apply to the other side of the unit-cube.

Finally, note that when one considers functions $g \in \mathbb{G} \backslash \mathbb{G}^{0}$, non transitive collective preferences trivially exist because the admissible set of individual preferences intersect with the non transitive regions. So, picking a uniform profile in this intersection leads to a non transitive collective preference (identical to each individual preference). The largest subclass of functions $g$ for which this phenomenon does not appear is precisely $\mathbb{G}^{0}$.

\section{The discrete case}

Propositions 2-5 can be compared with the results contained in Proposition 1 by Llamazares et al. [19]. Point (i) in Proposition 1 shows that for each $g \in \mathbb{G}$, there does not exist a threshold $k \in$ $\left[0, n-1\left[\right.\right.$ such that $P_{k}$ is transitive for each profile of reciprocal preference relations. Propositions 2 and 3 characterize the regions for which $P_{\varepsilon}$ is non transitive for $g=0.5$. Propositions 4 and 5 characterize these regions for $g \in \mathbb{G}^{0}$. On the one hand, our results reveal that these regions are non-empty for $\varepsilon \in[0,0.5[$, which is consistent with result (i) of Proposition 1 . On the other hand, point (ii) of Proposition 1 establishes that for each $g \in \mathbb{G}$ greater than or equal to the arithmetic mean and each threshold $k \in\left[n-1, n\left[, P_{k}\right.\right.$ is transitive for each profile of reciprocal preference relations. Point 3. of Proposition 5 differs from results (ii) and (iii) of Proposition 1 on two aspects. First, point 3. of Proposition 5 indicates that the regions $R_{5}$ and $R_{5}^{*}$ for which $P_{\varepsilon}$ is non transitive are non empty, though their volume is very small, for $\varepsilon$ close to 0.5 . Furthermore, this remains true even if $g$ is greater than or equal the arithmetic mean, e.g. $g=$ min, whereas result (iii) of Proposition 1 indicates that result (ii) holds only if $g$ is greater than or equal the arithmetic mean off the main diagonal. These differences are only due to the fact that Llamazares et al. [19] consider a finite and not necessarily large population. To obtain a clearer understanding of these differences, it is instructive to provide a geometric proof of the results contained in Proposition 1 by using one more time our representation of the problem.

Figure 11 displays the intersection of Figure 8 with the plane of equation $Q(a, b)=Q(b, c)$, a plane that cuts the cube into two along the main diagonal. This figure has been drawn with $g$ being the arithmetic mean. 

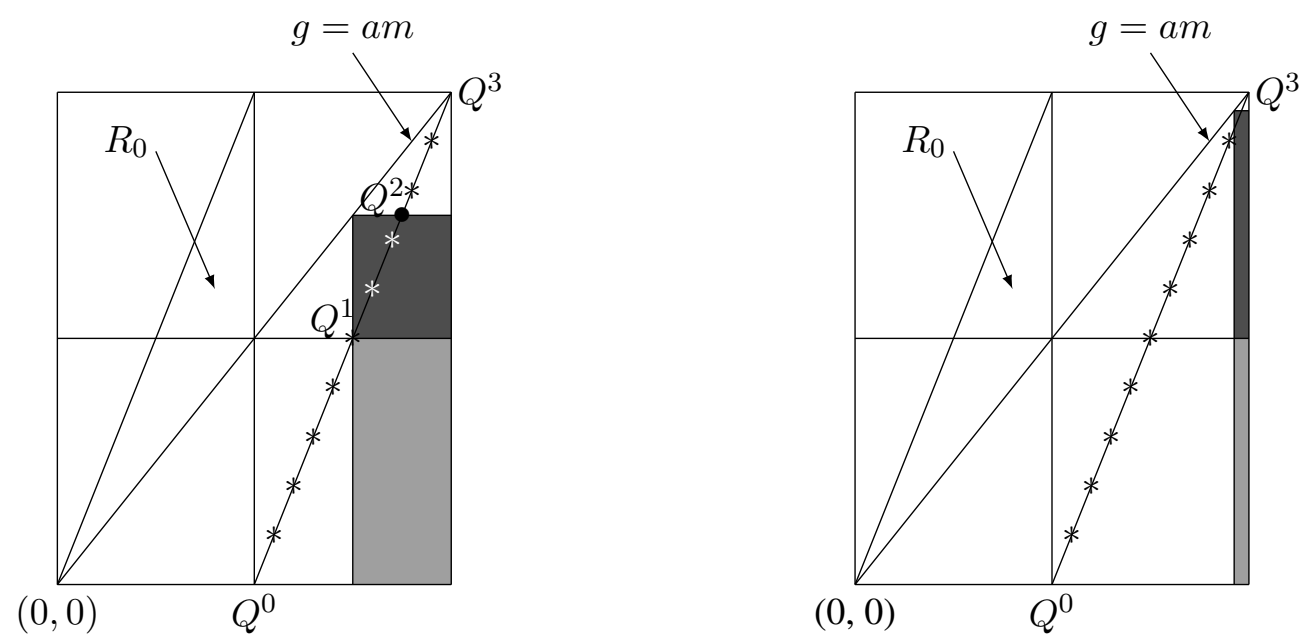

Figure 11: Geometric representation of results (i)-(ii) in Proposition 1 by Llamazares et al. [19]. This figure is drawn for $g=a m, \varepsilon=k /(2 n)=0.25$ where $n=10$ and $k=5$ (left-hand side), and $\varepsilon=k /(2 n)=0.475$ where $n=10$ and $k=9.5$ (right-hand side).

The key region is the intersection between the region of non transitivity of $P_{\varepsilon}$ and the region $R_{0}$. Consider the segment $\left[Q^{0} Q^{3}\right]$, where $Q^{0}=(0.5,0.5,0)$ and $Q^{3}=(1,1,1)$. The segment $\left[Q^{0} Q^{3}\right]$ always intersects the region of non-transitivity of $P_{\varepsilon}$. The intersection of the segment $\left[Q^{0} Q^{3}\right]$ with the region of non transitivity is given by the segment $\left[Q^{1} Q^{2}\right]$. Let us first compute the coordinates of point $Q^{2}$. By definition of $D_{2}$, the third coordinate of point $Q^{2}$ contained in the region of non transitivity verifies: $Q^{2}(a, c)=0.5+\varepsilon$. This point also lies both on the plane of equation (16) defining $R_{0}$ and, by construction, on the plane of equation $Q(a, b)=Q(b, c)$. From this, we obtain:

$$
Q^{2}(a, b)=Q^{2}(b, c)=\frac{3}{4}+\frac{\varepsilon}{2}, \text { and } Q^{2}(a, c)=0.5+\varepsilon .
$$

We now turn to the computation of the coordinates of point $Q^{1}$. Point $Q^{1}$ lies on the frontier of the parallelepiped $D_{2}$ defined in the section (3.1) and on the plane of equation (16). From this, we obtain:

$$
Q^{1}(a, b)=Q^{1}(b, c)=0.5+\varepsilon, \text { and } Q^{1}(a, c)=2 \varepsilon .
$$

Llamazares et al. [19] consider the discrete case where the population is finite and not necessarily large. In this context, consider that $l \in\{1, \ldots, n\}$ voters have a reciprocal preference relation given by $Q^{0}$, and $n-l$ voters have a reciprocal preference relation given by $Q^{3}$, and compute the collective preference relation $Q$ given by:

$$
Q=\frac{(n-l)}{n} Q^{3}+\frac{l}{n} Q^{0} .
$$

The intensity $Q(a, b)=Q(b, c)$ of $Q$ is given by the discrete convex combination:

$$
\frac{(n-l) \times 1}{n}+\frac{l \times 1}{2 n}=1-\frac{l}{2 n} .
$$

The intensity $Q(a, c)$ of the resulting collective preference relation $Q$ is given by the discrete convex combination:

$$
\frac{(n-l) \times 1+l \times 0}{n}=1-\frac{l}{n} .
$$


The collective preference relation $Q$ lies on the region of non transitivity if it belongs to the segment $\left[Q^{1} Q^{2}\right]$ and satisfies (8), that is if:

$$
0.5+\varepsilon<1-\frac{l}{2 n} \leq 0.75+\frac{\varepsilon}{2},
$$

and

$$
2 \varepsilon \leq 1-\frac{l}{n} \leq 0.5+\varepsilon .
$$

From (21) and (22) and the fact that $\varepsilon=k /(2 n)$, one obtains:

$$
\frac{n-k}{2} \leq l<n-k \text {. }
$$

We distinguish two cases.

Case 1: $k<n-1$. There are

$$
(n-k)-\left\lceil\frac{n-k}{2}\right\rceil
$$

values of $l$ satisfying condition (23). These values are represented by a white $*$ in the left-hand side of Figure 11 for $n=10$ and $k=5$. This construction is used in the proof of result (i) in Proposition 1 to show that $P_{k}$ cannot be transitive for all profiles of reciprocal preference relations.

Case 2: $k \geq n-1$. There is no $l \in\{1, \ldots, n\}$ satisfying condition (23). Indeed, for $k \geq n-1$, condition (23) forces $l<1$, which is not allowed. More precisely, if we consider the discrete convex combination between $Q^{0}$ and $Q^{3}$ such that $l=1$, then, by (19), the resulting collective preference relation belongs to $\left[Q^{1} Q^{2}\right]$ and satisfies (8) if:

$$
1-\frac{1}{2 n}>0.5+\frac{k}{2 n} \Longrightarrow k<n-1,
$$

which contradicts the initial hypothesis. This means than the resulting collective preference relation either coincides with $Q^{1}$ or lies outside the segment $\left[Q^{1} Q^{2}\right]$. Consequently, all other points that one can obtain by a discrete convex combination between $Q^{0}$ and $Q^{3}$ lie outside the segment $\left[Q^{1} Q^{2}\right]$. Such a situation is depicted in the right-hand side of Figure 11 for $n=10$ and $k=9.5$. This provides a geometric interpretation of why the argument developed for Case 1 fails when $k \in[n-1, n[$ and $g$ is greater than or equal to the arithmetic mean. This situation can not appear when the population is arbitrarily large and $g \in \mathbb{G}^{0}$ because the construction of non admissible collective preferences is always possible following Case 1. Result (ii) in Proposition 1 establishing that $P_{k}$ is transitive for all profiles of reciprocal preference relations when $k \in[n-1, n[$ and $g$ is greater than or equal to the arithmetic mean, is thus obtained by using an alternative argument. It consists of observing that individual preferences leading to a collective preference such that $a P_{k} b$ and $b P_{k} c$ necessarily lie in $R \cap[0.5,1]^{3}$, and that the convex hull of $R \cap[0.5,1]^{3}$ has an empty intersection with $G_{0} \cup G_{2} \cup D_{2}$.

On the contrary, when $g$ lies below the arithmetic mean off the main diagonal, i.e. when for each $(x, y) \in[0.5,1]^{2}$ such that $x \neq y$, we have $\left.g(x, y)<(x+y) / 2\right)$, it is possible to construct a convex combination of individual reciprocal preferences which lies in $G_{0} \cup G_{2} \cup D_{2}$. This is the case, for instance, when $g=\min$. This situation corresponds to result (iii) in Proposition 1. Such a construction can be done as follows: assume for the sake of simplicity that $n$ is even. Given a value of $k$, pick any $\alpha>0$ such that:

$$
0.5 \leq 0.5+\frac{k}{2 n}-\alpha \leq 0.5+\frac{k}{2 n}+\alpha \leq 1 .
$$


Set

$$
x=0.5+\frac{k}{2 n}-\alpha, \quad y=0.5+\frac{k}{2 n}+\alpha .
$$

In this way, we have:

$$
(x, y) \in[0.5,1]^{2}, x \neq y \text { and } \frac{x+y}{2}=0.5+\frac{k}{2 n} .
$$

Because $g$ is assumed to lie below the arithmetic mean, we have $g(x, y)=g(y, x)<(x+y) / 2$. Then, by continuity of $g$, there exists $\nu>0$ such that:

$$
g(x+\nu, y+\nu)=g(y+\nu, x+\nu)<\frac{x+y}{2} .
$$

Consider the individual reciprocal preferences $(x+\nu, y+\nu, g(x+\nu, y+\nu))$ and $(y+\nu, x+$ $\nu, g(y+\nu, x+\nu))$. Assume that one-half of the population has individual reciprocal preferences of the first type and the remaining agents have individual reciprocal preferences of the second type. Then, after a normalization, one obtains a collective outcome equal to:

$$
\left(\frac{x+y}{2}+\nu, \frac{x+y}{2}+\nu, g(x+\nu, y+\nu)\right) .
$$

The corresponding relation $P_{k}$ is not transitive since it holds that:

$$
\frac{x+y}{2}+\nu>\frac{x+y}{2}=0.5+\frac{k}{2 n}
$$

for the first two coordinates, and

$$
g(x+\nu, y+\nu)<\frac{x+y}{2}=0.5+\frac{k}{2 n} .
$$

\section{Conclusion}

This paper contains two new contributions. The first contribution aims at showing that the proof providing the necessary and sufficient conditions under which the majority based on difference in support is non transitive can be simplified. Instead of using algebraic tools, as in Llamazares et al. [19], we have demonstrated that this proof can be carried out with simple geometric tools. The second contribution concerns the regions for which the majority based on difference in support is non transitive. As a function of the model parameter $\varepsilon$, and for each kind of $g$-stochastic transitivity, we characterize the shape of the regions for which $P_{\epsilon}$ is non transitive when the electorate is large. As shown in Section 4, the geometric analysis proves also useful for a small electorate.

In the same manner as for the case of the non transitivity of $P_{\varepsilon}$, other types of inconsistencies of the majority based on difference in support can be studied under other points of view. A possible route can concern the undesirable collective preference defined by $a P_{\varepsilon} b, b I_{\varepsilon} c$ and $a I_{\varepsilon} c$. Another route can concern the cyclicity of majority based on difference in support: instead of the non transitivity of $P_{\varepsilon}$, we could look at the situations defined by $a P_{\varepsilon} b, b P_{\varepsilon} c$ and $c P_{\varepsilon} a$. Many other possibilities can also be considered using the same geometric approach.

\section{References}

[1] C.R. Barrett, P.K. Pattanaik, and M. Salles. On the structure of fuzzy social welfare functions. Fuzzy Sets and Systems, 19:1-10, 1986. 
[2] K. Basu. Fuzzy revealed preference theory. Journal of Economic Theory, 32:212-227, 1984.

[3] W.D. Cook and M. Kress. Ordinal ranking with intensity of preference. Management Science, 31:26-32, 1985.

[4] M. Dasgupta and R. Deb. Transitivity and fuzzy preferences. Social Choice ans Welfare, 13(3):305-318, 1996.

[5] B. De Baets and H. De Meyer. Transitivity frameworks for reciprocal relations: cycletransitivity versus FG-transitivity. Fuzzy Sets and Systems, 152:249-270, 2005.

[6] B. De Baets, H. De Meyer, B. De Schuymer, and S. Jenei. Cyclic evaluation of transitivity of reciprocal relations. Social Choice and Welfare, 26:217-238, 2006.

[7] D. Dubois and H. Prade. Fuzzy sets and systems: Theory and applications. mathematics in science and engineering. Academic Press, New York, 1980.

[8] J.L. García-Lapresta. A general class of simple majority decision rules based on linguistic opinions. Information Sciences, 176:352-365, 2006.

[9] J.L. García-Lapresta and B. Llamazares. Majority decisions based on difference of votes. Journal of Mathematical Economics, 35:463-481, 2001.

[10] J.L. García-Lapresta and B. Llamazares. Preference intensities and majority decisions based on difference of support between alternatives. Group Decision and Negotiation, 19:527-542, 2010.

[11] J.L. García-Lapresta and L.C. Meneses. Individual-valued preferences and their aggregation: Consistency analysis in a real case. Fuzzy Sets and Systems, 151(2):269-284, 2005.

[12] W.V Gehrlein and D. Lepelley. The value of research based on simple assumptions about voters' preferences. In: Electoral Systems: Paradoxes, Assumptions and Procedures, Publisher: Springer-Verlag, Editors: DS Felsenthal, M Machover, pages 173-200, 2012.

[13] N. Houy. Some further characterizations for the forgotten voting rules. Mathematical Social Sciences, 53:111-121, 2007.

[14] B. Llamazares. Simple and absolute special majorities generated by OWA operators. European Journal of Operational Research, 158:707-720, 2004.

[15] B. Llamazares. The forgotten decision rules: Majority rules based on difference of votes. Mathematical Social Sciences, 51:311-326, 2006.

[16] B. Llamazares. Choosing OWA operator weights in the field of Social Choice. Information Sciences, 177:4745-4756, 2007.

[17] B. Llamazares and J.L. García-Lapresta. Voting systems generated by quasiarithmetic means and OWA operators. In: Fodor, J., De Baets, B. (Eds.), Principles of Fuzzy Preference Modelling and Decision Making. Academia Press, Ghent, pages 195-213, 2003.

[18] B. Llamazares and J.L. García-Lapresta. Extension of some voting systems to the field of gradual preferences. In: Bustince, H., Herrera, F., Montero, J. (Eds.), Fuzzy Sets and Their Extensions: Representation, Aggregation and Models. Springer-Verlag, Berlin, pages 292310, 2008. 
[19] B. Llamazares, P. Perez-Asurmendi, and J.L. García-Lapresta. Collective transitivity in majorities based on difference in support. Fuzzy Sets and Systems, 216:3-15, 2013.

[20] I. McLean and A.B. Urken. Classics of social choice. Ann Arbor, The University of Michigan Press, 1995.

[21] B.L. Meek. A transferable voting system including intensity of preference. Mathématique et Sciences Humaines, 50:23-29, 1975.

[22] J.I. Morales. Memoria matemática sobre el cálculo de la opinion en las elecciones. Imprenta Real, Madrid, 1797.

[23] H. Nurmi. Approaches to collective decision making with fuzzy preference relations. Fuzzy Sets and Systems, 6:249-259, 1981.

[24] H. Nurmi. Fuzzy social choice: a selective retrospect. Soft Computing, 12:281-288, 2008.

[25] S.A. Orlovsky. Decision making with a fuzzy preference relation. Fuzzy Sets and Systems, 1:155-167, 1978.

[26] S.V. Ovchinnikov. Structure of fuzzy binary relations. Fuzzy Sets and Systems, 6:169-195, 1981.

[27] D.G. Saari. Geometry of voting. Springer, Berlin, Heidelberg, New York, 1994.

[28] D.G. Saari. Basic geometry of voting. Springer, Berlin, Heidelberg, New York, 1995.

[29] A.K. Sen. Collective Choice and Social Welfare. Holden-Day, San Francisco., 1970.

[30] T. Tanino. Fuzzy preference orderings in group decision making. Fuzzy Sets and Systems, 12:117-131, 1984.

[31] L.A. Zadeh. Similarity relations and fuzzy orderings. Information Sciences, 3(2):177-200, 1971. 